ISSN: 2641-1652

\title{
Primary Liver Cancer in Adults
}

\author{
Mirta D Ambra* \\ FWAMS MD, Ministry of Health of the Nation, Argentina

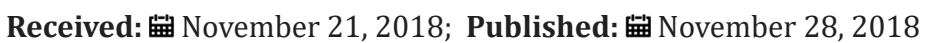 \\ *Corresponding author: Mirta D Ambra, FWAMS MD, Ministry of Health of the Nation, Argentina
}

\section{Case Report}

Liver cancer includes two main types: hepatocellular carcinoma (HCC) and intrahepatic bile duct cancer.

Incidence and mortality. Calculation of the number of new cases and deaths due to liver and intrahepatic bile duct cancer in the United States in 2018:

I. New cases: 42220 .

II. Deaths: 30200.

HCC is relatively rare in the United States, although its incidence is increasing, especially in regard to the spread of infection with the hepatitis C virus (HCV). HCC is the most common solid tumor in the world and the third leading cause of cancer-related deaths.

Anatomical Characteristics: Anatomy of the liver. The liver is in the upper part of the abdomen, near the stomach, the intestines, the gall bladder and the pancreas. The liver has a right lobe and a left lobe.

Risk Factor's: The etiology of HCC is probably multifactorial. The following factors may increase the risk of CHC: Infection with the hepatitis B virus (HBV) and infection with the hepatitis $\mathrm{C}$ virus (HCV): HBV and HCV infections seem to be the most important causes of HCC in the world. Chronic HBV infection is the main cause of HCC in Asia and Africa. HCV infection is the main cause of HCC in North America, Europe and Japan. The annual incidence of HCC in HBV carriers is 0.5 to $1 \%$ per year in non- cirrhotic patients and $2.5 \%$ per year in cirrhotic patients. The relative risk of HCC is 100 (that is, HBV carriers are 100 times more likely to have HCC than people who are not infected) [1] In a single prospective population study that included 12008 patients, the presence of positivity for anti-HCV increased the risk of HCC twenty-fold more than in antiHCV negative people. HCC can occur in fibrosis patients on bridges, infected with HCV, even in the absence of overt cirrhosis. However, the highest risk is that of patients with confirmed cirrhosis related to $\mathrm{HCV}$, which has an annual incidence rate of 2 to $8 \%$. [2]

Alcoholic Cirrhosis: Several reports indicate that alcoholic cirrhosis is a risk factor for HCC. However, the true incidence of HCC in alcoholic cirrhosis is not known because most of the epidemiological reports on this subject were published prior to the identification of HCV [3].

Metabolic Syndrome: Risk factors related to the metabolic syndrome, such as insulin resistance, hypertension, dyslipidemia and obesity were recognized as possible causes of nonalcoholic fatty liver disease, cirrhosis and HCC. However, to date there are no studies in which a fairly large group of patients was followed long enough to describe the incidence of HCC caused by the metabolic syndrome.

Biliary Cirrhosis: The incidence of stage IV HCC due to primary biliary cirrhosis is approximately the same as that of cirrhosis resulting from hepatitis $\mathrm{C}$.

Chronic Hepatic Injury: Chronic liver injury is likely to increase the risk of HCC, especially in patients with cirrhosis. The cumulative 5 -year risk of HCC in patients with cirrhosis ranges from 5\% to $30 \%$ and depends on the etiology (the highest among people with HCV infection), the region or the ethnic group (the highest among Asians). and the stage of cirrhosis [Level of evidence: 3iii]

Hemochromatosis: Hemochromatosis is an important risk factor for HCC and has a relative risk increase twenty times higher than that of the normal population

Aflatoxin B1: Aflatoxin B1 is produced by fungi of the genus Aspergillus and is a common contaminant of cereals, nuts and vegetables in some parts of Asia and Africa. Aflatoxin B1 has also been indicated as a cofactor in the etiology of primary liver cancer in HBV carriers because it increases the neoplastic risk to triple Screening tests.

\section{Diagnostic Factors}

For lesions smaller than $1 \mathrm{~cm}$ and found during screening in patients at high risk of HCC, no further diagnostic evaluation is needed because most of these lesions will be cirrhotic nodules instead of HCC. The common surveillance strategy is a careful followup at three-month intervals with the use of the same technique with which the presence of the lesions was documented for the first 
time. For patients with liver lesions larger than $1 \mathrm{~cm}$ who are at risk of HCC, a diagnosis should be made. The tests needed to diagnose an HCC may include radiology, biopsy, or both.

\section{Diagnostic Imaging}

For patients with cirrhosis, liver disease or other risk factors for HCC, and lesions larger than $1 \mathrm{~cm}$, optimized triple phase contrast studies (dynamic computed tomography (CT) or magnetic resonance imaging [MRI]) can be used. to establish a diagnosis of CHC. MRI in different phases of perfusion serve to evaluate the entire liver. After the controlled administration of a contrast material intravenously, images of the arterial and venous perfusion phases are taken. During the arterial phase of the study, HCC stands out more strongly than the surrounding liver because the arterial blood in the liver is diluted in the venous blood that does not contain contrast, while the HCC contains only arterial blood. In the venous phase, HCC stands out less than the surrounding liver (which is known as venous lavage of HCC) because the arterial blood flowing through the lesion no longer contains contrast; however, the portal blood in the liver now contains contrast.

\section{Liver Biopsy}

A liver biopsy can be performed when the diagnosis of HCC is not established by a dynamic imaging technique (three-phase CT or MRI) of liver lesions of $1 \mathrm{~cm}$ or more in high-risk patients.

\section{Concentrations of Alpha-Fetoprotein}

Alpha-fetoprotein (AFP) is not sensitive or specific enough to be used as a diagnostic assay. AFP may be elevated in intrahepatic cholangiocarcinoma and in some cases in which there is metastasis of colon cancer. The finding of a mass in the liver with a high concentration of AFP does not automatically indicate an HCC However, if the concentration of AFP is high, it can be used to monitor recurrence.

\section{Forecast}

Little is known about the natural history of early tumors because most patients with HCC receive treatment. However, in the oldest reports survival rates of 13 to $21 \%$ were described without any specific treatment. Currently, only 10 to $23 \%$ of HCC patients may be eligible for surgical treatment with curative intent. 5-year overall survival (OS) rates for early-stage HCC patients who undergo transplantation range from $44 \%$ to $78 \%$; for patients who undergo liver resection, the OS rate ranges from $27 \%$ to $70 \%$. Liver transplantation, surgical resection and ablation offer high rates of complete response and a chance of cure in early-stage HCC patients [2]. There is greater knowledge about the natural evolution of HCC in advanced stage. Patients with advanced disease without treatment usually survive less than 6 months. In 25 randomized clinical trials, the survival rate of untreated patients ranged from 10 to $72 \%$ at 1 year, and from 8 to $50 \%$ at 2 years. Unlike most patients with solid tumors, the prognosis of HCC patients is affected not only by the stage of the tumor at the time of the tumor at the time of its presentation, but also by the underlying hepatic function. The main prognostic factors that guide the selection of treatment are the following:

A) Anatomical extension of the tumor (i.e., tumor size, number of lesions, presence of vascular invasion and extrahepatic dissemination) [4-6].

\section{Functional State}

Hepatic functional reserve according to the Child-Pugh score.

\section{Conclusion}

Cases of liver cancer have been on the rise since the 1980s. Before 2016, the American Cancer Society estimated that 39,230 new cases and 27,170 people would die as a result of it in the United States. Liver cancer is the fifth leading cause of cancer-related deaths in the United States. It has the mortality rate with the fastest growth of all cancers in the country. Currently, liver cancer has a poor prognosis since most cases are not detected in early stages.

\section{References}

1. Beasley RP, Hwang LY, Lin CC (1981) Hepatocellular carcinoma and hepatitis B virus. A prospective study of 22707 men in Taiwan. Lancet 2 (8256): 1129-1133.

2. Bruix J, Sherman M (2011) American Association for the Study of Liver Diseases: Management of hepatocellular carcinoma: an update. Hepatology 53(3): 1020-1022.

3. Forner A, Llovet JM, Bruix J (2012) Hepatocellular carcinoma. Lancet 379(9822): 1245-1255.

4. American Cancer Society (2018) Cancer Facts and Figures 2018. Atlanta, Ga: American Cancer Society, Available onlineNotificación de salida.

5. Altekruse SF, McGlynn KA, Reichman ME (2009) Hepatocellular carcinoma incidence, mortality, and survival trends in the United States from 1975 to 2005. J Clin Oncol 27(9): 1485-1491.

6. Bosch FX, Ribes J, Borràs J (1999) Epidemiology of primary liver cancer. Semin Liver Dis 19(3): 271-285. 
(c) (P) This work is licensed under Creative

To Submit Your Article Click Here: Submit Article

DOI: $10.32474 /$ CTGH.2018.01.000122

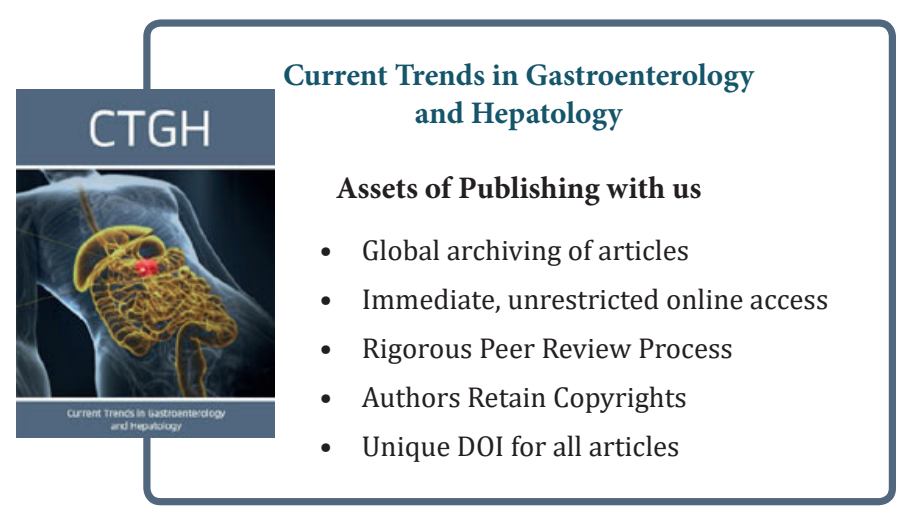

